\title{
Hypotaurine is an Energy-Saving Hepatoprotective Compound against Ischemia-Reperfusion Injury of the Rat Liver
}

\author{
Tadayuki Sakuragawa $^{1}$, Takako Hishiki ${ }^{1}$, Yuki Ueno ${ }^{1}$, Satsuki Ikeda ${ }^{1}$, Tomoyoshi Soga ${ }^{2}$, \\ Ayako Yachie-Kinoshita', Mayumi Kajimura ${ }^{1}$ and Makoto Suematsu,* \\ ${ }^{1}$ Department of Biochemistry and Integrative Medical Biology, School of Medicine, Keio University, \\ Tokyo 160-8582, Japan \\ ${ }_{2}^{2}$ Institute for Advanced Biosciences, Keio University, Tsuruoka City 997-0017, Japan
}

Received 29 September, 2009; Accepted 16 October, 2009

\begin{abstract}
Summary Metabolome analyses assisted by capillary electrophoresis-mass spectrometry (CE-MS) have allowed us to systematically grasp changes in small molecular metabolites under disease conditions. We applied CE-MS to mine out biomarkers in hepatic ischemiareperfusion. Rat livers were exposed to ischemia by clamping of the portal inlet followed by reperfusion. Metabolomic profiling revealed that $I$ contents of taurine in liver and plasma were significantly increased. Of interest is an elevation of hypotaurine, collectively suggesting significance of hypotaurine/taurine in post-ischemic responses. Considering the anti-oxidative capacity of hypotaurine, we examined if supplementation of the compound or its precursor amino acids could affect hepatocellular viability and contents of taurine in liver and plasma. Administration of hypotaurine, $\mathbf{N}$-acetylcysteine or methionine upon reperfusion comparablly attenuated the post-ischemic hepatocellular injury but with different metabolomic profiling among groups: rats treated with methionine or $\mathrm{N}$-acetylcysteine but not those treated with hypotaurine, exhibited significant elevation of hepatic lactate generation without notable recovery of the energy charge. Furthermore, the group treated with hypotaurine exhibited elevation of the plasma taurine, suggesting that the exogenously administered compound was utilized as an antioxidant. These results suggest that taurine serves as a surrogate marker for ischemia-reperfusion indicating effectiveness of hypotaurine as an energy-saving hepatoprotective amino acid.
\end{abstract}

Key Words: metabolome, CE-MS, hypotaurine, taurine, reperfusion injury

\section{Introduction}

Ischemia-reperfusion injury has been proposed to result from a burst of reactive oxygen species (ROS) generated upon reperfusion, and administration of antioxidants at the

\footnotetext{
*To whom correspondence should be addressed.

Tel: +81-3-5363-3753 Fax: +81-3-5363-3466

E-mail: gasbiology@zb.keio.jp

Grant Supports: This study was supported by Grain-in-Aid for Creative Science Research 17GS0419. Global COE Program for Metabolomics Systems Biology from MEXT and ERATO Gas Biology Project from the Japan Science and Technology Agency.
}

onset of reperfusion diminished the post-ischemic injury [1]. There are multiple resources of ROS upon reperfusion; endothelial cells, leukocytes and mitochondria mainly in parenchymal cells that constitute major cellular fraction for $\mathrm{O}_{2}$ consumption $[2,3]$. In case of the reperfusion injury of the liver, site-specific delivery of superoxide dismutase (SOD) to endothelial cells serves as a potentially effective strategy to attenuate the injury [4]. On the other hand, only limited methods have been available to attenuate ROS from the mitochondrial origin. In other words, there is a paucity of availability of small molecular anti-oxidative compounds that can directly access the site of ROS generation in hepatocytes. 
In this context, application of N-acetylcysteine (NAC) might serve as one of the important compounds widely used to attenuate such an intracellular resource of ROS [5]. This compound is believed to enter the cells and catalyzed to produce cysteine as a precursor of reduced glutathione (GSH) or as a direct antioxidant by itself [5, 6]. However, little evidence has been provided for evidence whether administration of NAC might alter metabolic systems as a whole to promote other beneficial effects besides scavenging ROS or any unidentified harmful effects on the reperfused liver.

To solve this question, we aimed to investigate alterations in small molecular metabolites in ischemia-reperfused rat liver, and to examine effects of administration of NAC on major metabolic pathways. To this end, we have utilized metabolome analyses based on capillary electrophoresis equipped with mass spectrometry (CE-MS) for systematic mining of biomarkers indicating the reperfusion injury of the liver [7-9]. The current results suggest that the postischemic liver exhibits remarkable increases in hepatic contents of hypotaurine and taurine after reperfusion, which coincided with a notable elevation of taurine in circulation. Furthermore, administration of NAC enrich two important antioxidant systems including both GSH and hypotaurine, while increasing hepatic lactate contents. To be noted is that a comparable dose of hypotaurine itself effectively attenuate the reperfusion injury independently of GSH enrichment, suggesting its crucial role against hepatic ischemia-reperfusion injury.

\section{Materials and Methods}

Animals and surgical operation for ischemia/reperfusion model

The experimental protocols herein described were approved by our institutional guidelines provided by the Animal Care Committee of Keio University School of Medicine. Male Wistar rats weighting 220 to $280 \mathrm{~g}$ were used. The rats were allowed free access to laboratory chow and tap water, and were fasted for $18 \mathrm{~h}$ prior to experiments. They were anesthetized with an intraperitoneal injection of sodium pentobarbital at $50 \mathrm{mg} / \mathrm{kg}$. The abdomen was opened by midline incision. To avoid effects of splanchnic blood congestion, we used the model of partial liver ischemia [10]. The ischemia was induced by selective clamping of branches of the portal vein and hepatic artery supplying the left lateral and left median liver lobes. After $1 \mathrm{~h}$ of the ischemia, the vascular clamp was released to establish reperfusion for 1,3 or $6 \mathrm{~h}$. After the removal of vascular clamp, the abdomen was closed in two layers. In sham operation, the abdomen was opened and the liver was manipulated similarly except for clamping of portal vein and hepatic artery.
After warm ischemia/reperfusion or sham operation, liver tissues were collected by snap frozen procedures [11]. Briefly, the portal vein was cannulated with a 16-gauge catheter, into which Krebs-Henseleit bicarbonate buffer cooled at $4^{\circ} \mathrm{C}$ was infused to remove blood. The aorta and inferior vena cava were dissected as the outflow tract. Liver samples were immediately frozen with liquid nitrogen and stored at $-40^{\circ} \mathrm{C}$ for later analysis. Five main protocols were employed in groups treated with 1-h ischemia followed by desired lengths of time for reperfusion: Livers in the first group were treated with an intraperitoneal injection of physiological saline as a vehicle at the start of reperfusion. In the second to the fifth groups, methionine (Met), NAC, hypotaurine (HTU) and taurine (TU) dissolved in physiological saline were intraperitoneally administered at the start of reperfusion at desired doses, respectively. The doses used in the current study were $1.5 \mathrm{mmol} / \mathrm{kg}$ [12-15]. These experiments were conducted to examine if hypotaurine and its precursor amino acids such as methionine or cysteine could improve post-ischemic injury of the liver through the antioxidative property of the compound.

\section{Metabolome analysis}

We performed metabolome analyses of tissue lysates collected from snap-frozen livers of rat using CE-MS according to our previous methods [7]. At desired periods of time for reperfusion, the abdominal incision was reopened for excising livers to be snap-frozen by liquid nitrogen and minced in cold methanol. In this study, we carried out determination of metabolites at 1,3 and $6 \mathrm{~h}$ after the start of reperfusion, and compared effects of the aforementioned amino acids on the reperfusion injury at $3 \mathrm{~h}$. The lysates served as samples for CE-MS analyses as reported previously $[7,8]$. In separate sets of experiments, liver samples collected from the groups were minced with $10 \%$ trichloroacetic acid at $4^{\circ} \mathrm{C}$ to measure cysteine and GSH through HPLC to confirm the data collected from CE-MS.

\section{Statistical analyses}

All data in this study were expressed as mean \pm SD. Differences in the mean values among the groups were analyzed by one-way ANOVA combined with Fisher's-type multiple comparison test. $p$ values smaller than 0.05 were considered statistically significant.

\section{Results}

\section{Metabolomic profiling of hepatic ischemia-reperfusion}

Table 1 indicated temporal alterations in metabolomic profiling of hepatic contents of metabolites. The data were normalized by the baseline values measured in the nonischemic control group, and expressed as fold changes as a function of time until $6 \mathrm{~h}$ after reperfusion. Among metabo- 
Table 1. Alterations in hepatic contents of metabolites after the post-ischemic reperfusion periods

\begin{tabular}{llll}
\hline $\begin{array}{l}\text { Contents of metabolites } \\
\text { (vs non-ischemic control) }\end{array}$ & $\mathrm{I} / \mathrm{R} 1 \mathrm{~h}$ & $\mathrm{I} / \mathrm{R} 3 \mathrm{~h}$ & $\mathrm{I} / \mathrm{R} 6 \mathrm{~h}$ \\
\hline Increased metabolites & & & \\
Taurine & $1.81 \pm 0.18^{*}$ & $2.61 \pm 0.22^{*}$ & $2.90 \pm 0.34^{*}$ \\
Hypotaurine & $1.08 \pm 0.30$ & $2.35 \pm 0.73$ & $3.71 \pm 1.53^{*}$ \\
Citrate & $1.28 \pm 0.17$ & $1.74 \pm 0.22^{*}$ & $0.99 \pm 0.23$ \\
CMP & $1.18 \pm 0.14$ & $1.55 \pm 0.14^{*}$ & $1.67 \pm 0.23^{*}$ \\
Adenosine & $1.37 \pm 0.22$ & $1.54 \pm 0.20$ & $1.48 \pm 0.39$ \\
Glutamate & $1.36 \pm 0.27$ & $1.46 \pm 0.17$ & $0.95 \pm 0.11$ \\
S-Adenosyl-L-homocysteine & $1.14 \pm 0.13$ & $1.45 \pm 0.14$ & $1.33 \pm 0.32$ \\
CDP & $0.94 \pm 0.12$ & $1.40 \pm 0.19^{*}$ & $1.60 \pm 0.06^{*}$ \\
S-Adenosyl-L-methionine & $0.89 \pm 0.12$ & $1.24 \pm 0.25$ & $1.00 \pm 0.22$ \\
CTP & $0.68 \pm 0.13$ & $1.14 \pm 0.14$ & $1.27 \pm 0.05$ \\
AMP & $1.16 \pm 0.13$ & $1.02 \pm 0.02$ & $1.06 \pm 0.08$ \\
NAD & $0.84 \pm 0.04^{*}$ & $1.00 \pm 0.04$ & $1.04 \pm 0.06$ \\
\hline Decreased metabolites & & & \\
ADP & $0.92 \pm 0.12$ & $0.97 \pm 0.06$ & $1.05 \pm 0.04$ \\
GMP & $1.13 \pm 0.08$ & $0.96 \pm 0.04$ & $0.96 \pm 0.15$ \\
NADH & $0.95 \pm 0.09$ & $0.94 \pm 0.06$ & $0.88 \pm 0.07$ \\
Glutathione & $0.67 \pm 0.09$ & $0.93 \pm 0.08$ & $0.62 \pm 0.08^{*}$ \\
GDP & $0.92 \pm 0.10$ & $0.90 \pm 0.03$ & $0.89 \pm 0.04$ \\
ATP & $0.65 \pm 0.10^{*}$ & $0.81 \pm 0.09$ & $0.92 \pm 0.04$ \\
Glycine & $1.05 \pm 0.12$ & $0.78 \pm 0.08$ & $0.80 \pm 0.15$ \\
GTP & $0.66 \pm 0.10^{*}$ & $0.76 \pm 0.07^{*}$ & $0.77 \pm 0.08$ \\
Methionine & $0.85 \pm 0.08$ & $0.74 \pm 0.19$ & $0.72 \pm 0.04$ \\
FAD & $0.86 \pm 0.02$ & $0.70 \pm 0.05^{*}$ & $0.75 \pm 0.03^{*}$ \\
NADPH & $0.86 \pm 0.14$ & $0.69 \pm 0.04^{*}$ & $0.65 \pm 0.08^{*}$ \\
CoA & $0.64 \pm 0.08$ & $0.68 \pm 0.07$ & $0.67 \pm 0.24$ \\
NADP & $0.79 \pm 0.03^{*}$ & $0.66 \pm 0.03^{*}$ & $0.69 \pm 0.05^{*}$ \\
Acetyl CoA & $0.65 \pm 0.23$ & $0.23 \pm 0.05^{*}$ & $0.62 \pm 0.17$ \\
\hline Not & & & \\
\hline
\end{tabular}

Note that metabolomic profiling of the metabolites post of ischemia-reperfusion as described in Materials and Methods. The data were expressed as those normalized by the average values of non-ischemic controls. Values are mean \pm SE of 4 separate experiments. ${ }^{*} p<0.05$ as compared with the control values.

lites indicating up-regulating responses after reperfusion, taurine and hypotaurine were those showing remarkable elevation by $2-3$ folds versus the baseline. On the other hand, those decreased significantly at $1 \mathrm{~h}$ after reperfusion were ATP and GTP; these metabolites were recovered partially during the later time points of measurements. On the other hand, other metabolites that were not listed in Table 1 did not exhibit any significant changes during the course of observations.

Exogenous methionine or $\mathrm{N}$-acetylcysteine attenuates postischemic liver injury

Fig. 1 illustrates the magnitudes of hepatocellular injury induced by 3-h reperfusion following ischemia which were judged by the leakage of ALT and GLDH into circulation and indicates the effects of post-ischemic administration of Met, NAC, HTU or TU. As shown, post-treatment of one of these reagents but not of TU significantly attenuated the release of the enzymes, indicating protective actions of these reagents against oxidative tissue injury by ischemiareperfusion.

Since results of metabolome analysis suggested notable changes in metabolism of hypotaurine and taurine, we next examined effects of Met, NAC or HTU on hepatic contents of taurine and its plasma concentration. Figure 2 illustrates results at $3 \mathrm{~h}$ after reperfusion in each group. As seen, the tissue contents of taurine increased by 2 folds versus the control level, and the treatment with Met, NAC or HTU did not greatly alter the taurine contents. On the other hand, the plasma concentration of taurine turned out to increase by 2.5 

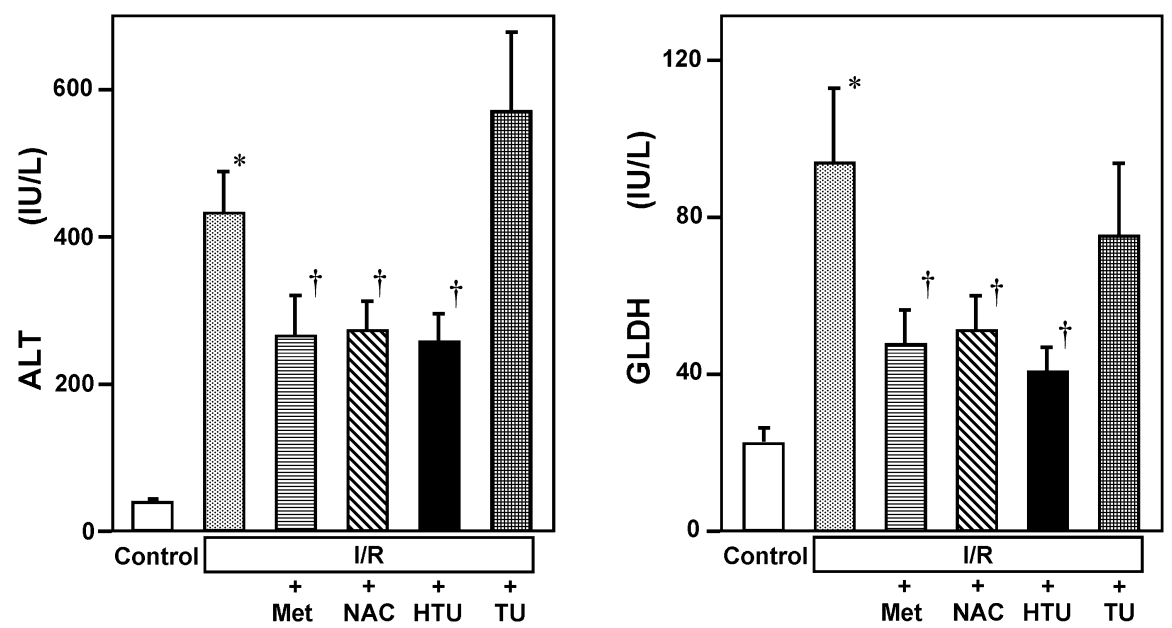

Fig. 1. Hepatoprotective effects of sulfur-containing amino acids such as methionine (Met), N-acetylcysteine (NAC), hypotaurine (HTU) and taurine (TU) on hepatocellular injury caused by 3-h reperfusion following 1-h ischemia in the rat liver. Control and $\mathrm{I} / \mathrm{R}$ indicate the sham-operated control group and that treated with ischemia-reperfusion, respectively. The aminoacids were administered immediately after the onset of reperfusion. Data indicate those measured at $3 \mathrm{~h}$ after reperfusion and were expressed as mean \pm SE of $5-15$ separate experiments. ${ }^{*} p<0.05$ vs the I/R group. ${ }^{\dagger} p<0.05$ vs the data in the I/R group treated with vehicles.
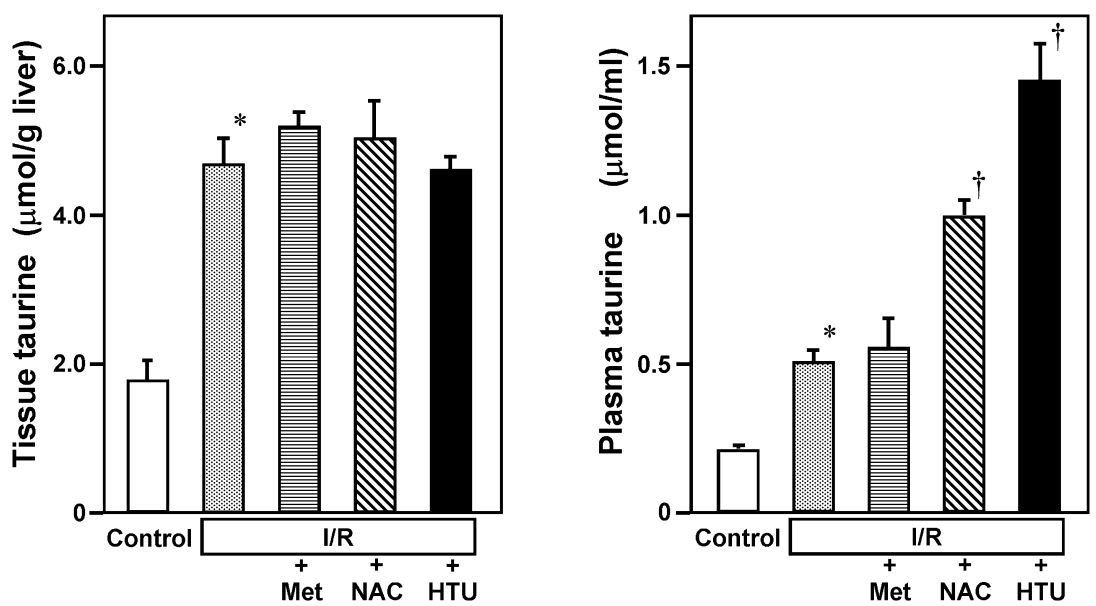

Fig. 2. Effects of methionine (Met), N-acetylcysteine (NAC) or hypotaurine (HTU) on hepatic contents and plasma concentrations of taurine. Data measured at $3 \mathrm{~h}$ after reperfusion were shown. Data were expressed as mean $\pm \mathrm{SE}$ of $5-8$ separate experiments. ${ }^{*} p<0.05$ as compared with the control group. ${ }^{\dagger} p<0.05$ vs the data in the I/R group.

folds in response to reperfusion, suggesting that taurine might serve as a surrogate marker indicating the hepatic reperfusion injury that is detectable in circulation.

To be noted is that administration of NAC or HTU in the model significantly increased plasma taurine concentration. Considering the results indicating that the NAC or HTU treatment did not increase the hepatic taurine contents, these results raised a possibility that administration of NAC or HTU might be metabolized in vivo to serve as a resource of de novo taurine synthesis to be excreted from the liver into circulation. To examine this hypothesis, we compared differences in metabolomic profiles among the experimental groups. Fig. 3 indicated a portion of results collected from CE-MS-based metabolome analyses that focused on sulfurcontaining amino acid metabolism. As seen, the group treated with Met indicated a remarkable elevation of Met, S-adenosylmethionine (SAM), and S-adenosylhomocysteine $(\mathrm{SAH})$ in remethylation cycle as well as cystathionine located at transsulfuration pathway. Other important changes were increases in hypotaurine and reduced form of GSH, suggesting that exogenous Met is utilized for enrichment of the anti-oxidative substrates in the post-ischemic liver.

Observation that supplementation of Met from the top of remethylation and transsulfuration pathways increased the 


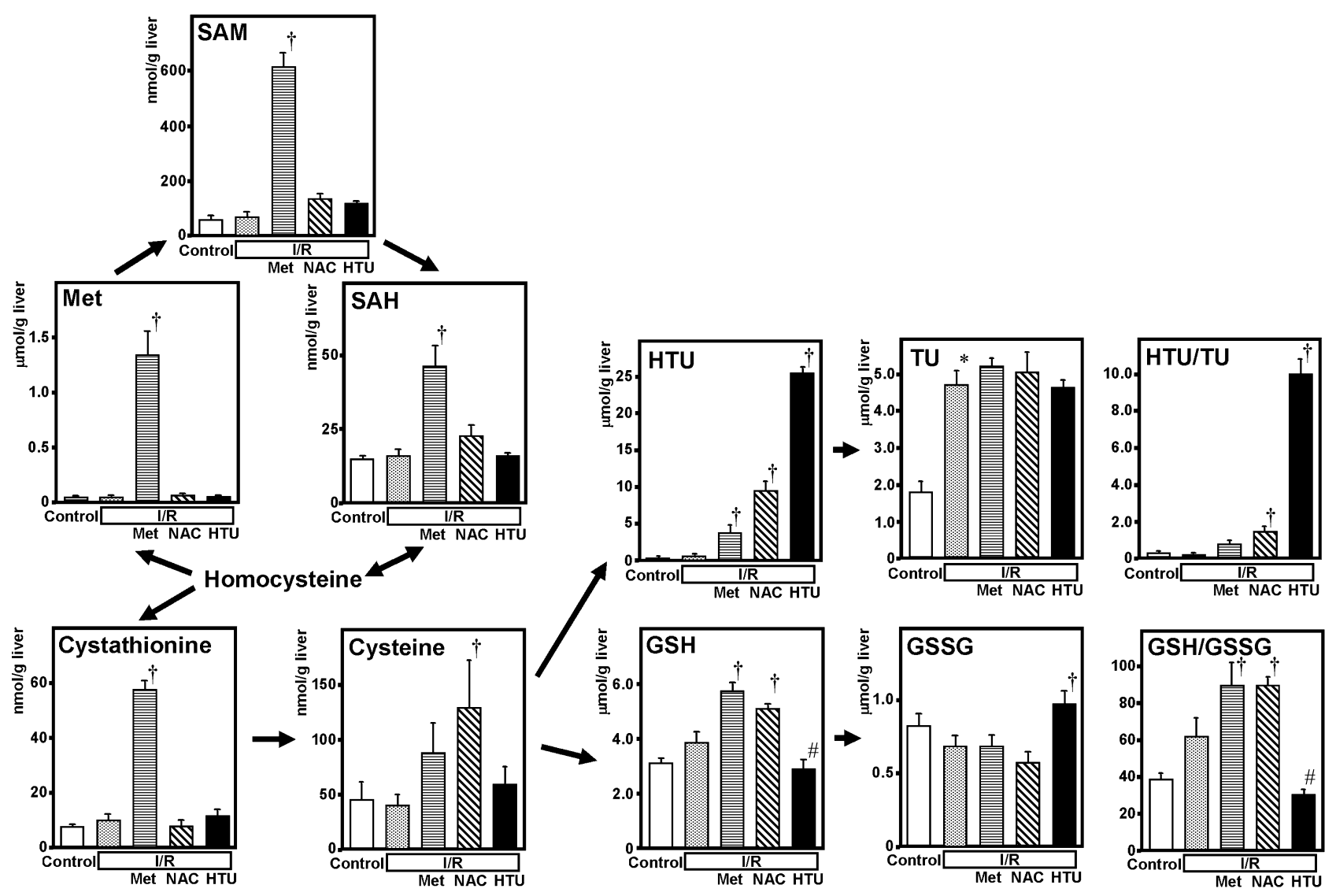

Fig. 3. Altertaions in metabolites gelongingto remethylation and transsulfuration pathways upon administration of sulfur-containing amino acids such as methionine (Met), N-acetylcysteine (NAC) and hypotaurine (HTU). SAM: S-adenosylmethionine, SAH: S-adenosylhomocysteine. Note that the protective effects of hypotaurine which was shown in Fig. 1 occur independently of GSH supplementation. Values are mean \pm SE of $5-8$ separate experiments. ${ }^{*} p<0.05$ vs the control. ${ }^{\dagger} p<0.05$ vs the I/R-treated group. ${ }^{\#} p<0.05$ vs the $\mathrm{I} / \mathrm{R}+$ Met group.

downstream antioxidants such as GSH and hypotaurine led us to speculate that exogenous administered NAC might also be converted to the two different antioxidants. As expected, the supplementation of NAC significantly increased both GSH and hypotaurine, but without altering contents of the upstream metabolites such as Met, SAM, SAH and cystathionine. Under the given dose, both Met and NAC resulted in comparable supplementation of GSH and HTU, being in good agreement with the results showing both supplements comparably attenuated the hepatocellular injury (Fig. 1).

\section{HTU attenuates the injury without enhancing the GSH system}

The current results showing the comparability of hepatoprotective effects between Met and NAC and their beneficial effects on increased contents of GSH and hypotaurine led us to inquire which antioxidant system might play a crucial role against hepatocellular injury caused by ischemiareperfusion of the liver. To answer to the inquiry, effects of the comparable dose of HTU were examined using the same protocol. As seen in Fig. 3, The HTU-treated liver exhibited significant elevation of the hepatic contents of HTU and accordingly, the ratio between HTU and TU. Taken together with the observation in Fig. 2 that the same treatment significantly increased plasma taurine concentration, these results suggest that exogenously administered HTU converted to TU to serve as an antioxidant to protect against the cell injury. Another important feature of the exogenous HTU administration that was not seen in the Met or NAC treatment was the absence of GSH elevation: In the HTU-treated liver, oxidized form of glutathione (GSSG) was significantly elevated versus other groups. This observation was consistent with a notion that, in the HTU-treated group, endogenous GSH is consumed as a result of oxidative stress without being supplied by the upstream substrates.

HTU but not Met nor NAC improves energy charge in the post-ischemic liver injury

Results of metabolome analysis displayed a distinct effect 

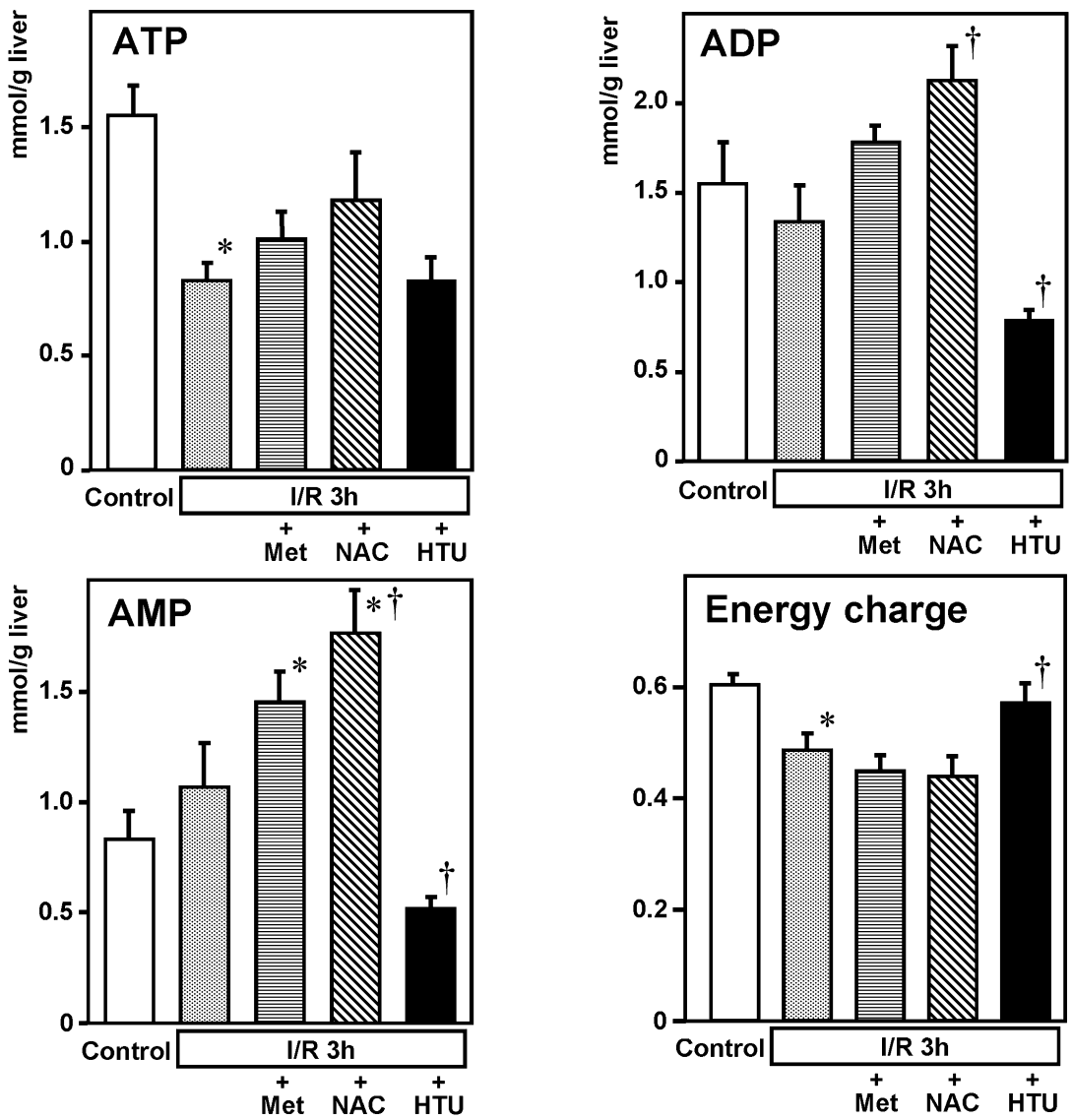

Fig. 4. Hepatic contents of ATP, ADP and AMP and energy charges measured at $3 \mathrm{~h}$ after the start of reperfusion. Note that the HTUtreated group significantly attenuated tissue contents of ADP and AMP, without lowering ATP in the post-ischemic liver, leading to a significant recovery of the energy charge. Values are mean \pm SE of $5-8$ separate experiments. ${ }^{*} p<0.05$ vs the control. ${ }^{\dagger} p<0.05$ vs the I/R-treated group.

of HTU from that of Met or NAC in a profile of nucleotide contents in the post-ischemic liver. Figure 4 illustrates the tissue contents of ATP, ADP and AMP and energy charges calculated from these data collected at $3 \mathrm{~h}$ after the start of reperfusion. As seen, treatment with Met or NAC did not improve contents of ATP, while increasing contents of ADP and AMP. As a result, the value of energy charge was not improved in these groups. On the other hand, The HTU treatment significantly attenuated tissue contents of ADP and AMP, while not altering the ATP contents in the postischemic liver, and resulted in a significant recovery of the energy charge value.

Treatment with Met or NAC but not with HTU aggravates lactate overproduction in the post-ischemic liver

Exogenously administered amino acids have been known to undergo transamination and oxidative deamination to produce carbohydrates that serve as substrates for tricarbonic acid (TCA) cycle and gluconeogenesis in the liver. Since the effects on energy charge were different between Met- or NAC- and HTU-treated post-ischemic livers, we compared the response of carbohydrate metabolism including glycolysis, gluconeogenesis and TCA cycle. As seen in Fig. 5, the group treated with 3-h reperfusion following the ischemia displayed a significant decrease in acetyl CoA and increases in citrate, succinate, malate and phosphoenolpyruvate (PEP). In the groups treated with Met, the decrease in acetyl CoA was significantly recovered, while that with NAC displayed a marked elevation of Acetyl CoA, presumably because of supplementation with the acetyl group from the compound. In the groups treated with Met or NAC, hepatic contents of lactate and fructose 6-phosphate were significantly elevated. On the other hand, the group treated with HTU did neither exhibit any remarkable changes in TCA substrates nor induce the elevation of lactate and fructose 6-phopsphate. These results suggest that HTU did neither trigger gluconeogenesis nor accelerate glycolysis in the post-ischemic liver tissue.

\section{Discussion}

The current study using CE-MS-based metabolome anal- 


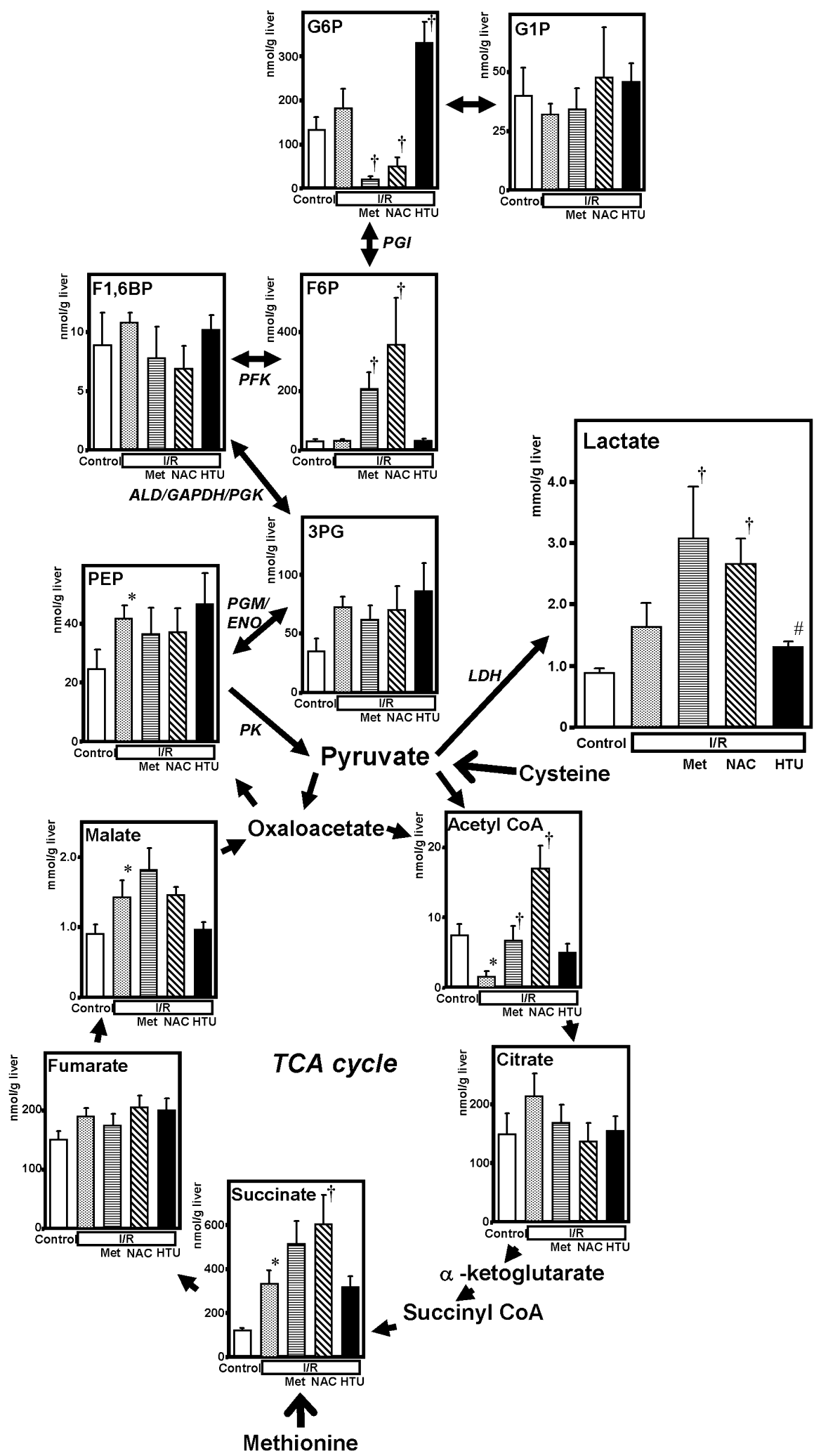

Fig. 5. Altertaions in carbohydrate metabolism in glycolysis/gluconeogenesis and TCA cycle upon administration of methionine (Met), $\mathrm{N}$-acetylcysteine (NAC) and hypotaurine (HTU) in the ischemia-reperfused rat liver. Note that the group treated with Met or NAC, but not that with HTU, exhibited significant elevation of lactate. Values are mean \pm SE of 5-8 separate experiments. ${ }^{*} p<0.05$ vs the control. ${ }^{\dagger} p<0.05$ vs the I/R-treated group. ${ }^{\#} p<0.05$ vs the $\mathrm{I} / \mathrm{R}+$ Met group. 
ysis first provided evidence that taurine serves as a surrogate marker indicating warm ischemia-reperfusion injury of the liver. Furthermore, the study revealed that administration of hypotaurine, the precursor of taurine, significantly attenuated the post-ischemic liver injury without aggravating energy metabolism and lactate overproduction. On the other hand, either NAC, a precursor of cysteine widely used for an antioxidant accessible to the intracellular space, or Met turned out to aggravate the energy charge values through incorporating into TCA cycle to be utilized as a substrate for gluconeogenesis, while these compounds exert their hepatoprotective ability against the post-ischemic insults. It is unknown why hypotaurine serves as an energy-saving antioxidant as compared with NAC. However, so far as judged from the data indicating effects of NAC on alterations in amounts of TCA substrates and gluconeogenesis, it is not unreasonable to speculate that, through being catalized into cysteine and acetate, the compound might provide acetyl $\mathrm{CoA}$ for the substrate and increases gluconeogenic substrates such as PEP and fructose 6-phosphate. Since gluconeogenesis is an energy-consuming process, such a hypothesis is in good agreement with the metabolome data that indicate accumulation of gluconeogenic products and decreased energy charge.

The present study also revealed that distinct role between GSH/GSSG and hypotaurine/taurine systems for antioxidative defense mechanisms in the liver. In the groups treated with Met or NAC, the post-ischemic liver increased contents of GSH through providing cysteine as the precursor. The ratio between GSH versus GSSG was well conserved in both Met-treated and NAC-treated groups. On the other hand the post-ischemic liver treated with hypotaurine, the GSH/GSSG ratio was significantly reduced presumably because of elevated oxidation of GSH into GSSH through a paucity of de novo supply of GSH during post-ischemic oxidative stress. Under these circumstances, however, exogenously administered hypotaurine appeared to be converted into taurine to exert its anti-oxidative property aginst the injury. Distinct from the GSH/GSSH system, hypotaurine seems to be a suicide antioxidative compound that is oxidized by ROS [16-18] to form taurine that is excreted through its transporter into the extracellular space. Because of such nature of the compound, the results of the present study suggest that effects of extracellularly administered hypotaurine could be estimated by monitoring the increase in plasma concentrations of taurine as seen in Fig. 2.

In the present study, metabolome analysis allowed us to reveal diverse effects of NAC. This compound has widely been used as a potent antioxidant both experimentally and clinically [6], and its major effect has been thought to be an increase in the GSH/GSSG ratio. So far as seen in the present results, once administered in vivo, NAC increases both GSH and hypotaurine to potentiate the ROS-scavenging capacity. In addition to such effects, the compound appeared to undergo deamination to produce pyruvate that accounts for substrate of varied carbohydrate products including lactate, TCA cycle products and PEP. In other words, an excess overload of gluconeogenic substrates upon exogenous administration of amino acids might jeopardize the energy state of the liver through energy-consuming gluconeogenesis. In this context, the current results suggest that hypotaurine serves as an energy-saving antioxidant protecting against post-ischemic oxidative injury.

\section{Acknowledgments}

This work was supported by Leading Project for Biosimulation and by Next Generation Supercomputing Sciences and Applications from RIKEN.

\section{Abbreviations}

ALD, aldolase; ALT, alanine transferase; CE-MS, capillary electrophoresis-mass spectrometry; ENO, enolase; F1,6BP, fructose 1,6-bisphosphate; F6P, fructose 6-phosphate; GAPDH, glyceraldehydephosphate dehydrogenase; GLDH, glutamate dehydrogenase; GSH, glutathione; GSSG, oxidized form of glutathione; G1P, glucose 1-phosphate; G6P, glucose 6-phosphate; HTU, hypotaurine; LDH, lactate dehydrogenase; Met, methionine; NAC, N-acetylcysteine; PEP, phosphoenolpyruvate; PFK, phosphofructokinase; 3PG, 3phosphoglycerate; PGI, phosphoglucoisomerase; PGK, phosphoglycerate kinase; PGM, phosphoglycerate mutase; $\mathrm{PK}$, pyruvate kinase; ROS, reactive oxygen species; SAH, $S$-adenosylhomocysteine; SAM, $S$-adenosylmethionine; SOD, superoxide dismutase; TU, taurine.

\section{References}

[1] Rosseland, C.M., Wierod, L., Oksvold, M.P., Werner, H., Ostvold, A.C., Thoresen, G.H., Paulsen, R.E., Huitfeldt, H.S., and Skarpen, E.: Cytoplasmic retention of peroxide-activated ERK provides survival in primary cultures of rat hepatocytes. Hepatology, 42, 200-207, 2005.

[2] Suzuki, H., Suematsu, M., Ishii, H., Kato, S., Miki, H., Mori, M., Ishimura, Y., Nishino, T., and Tsuchiya, M.: Prostaglandin E1 abrogates early reductive stress and zonespecific paradoxical oxidative injury in hypoperfused rat liver. J. Clin. Invest., 93, 155-164, 1994.

[3] Jaeschke, H.: Molecular mechanisms of hepatic ischemiareperfusion injury and preconditioning. Am. J. Physiol. Gastrointest. Liver Physiol., 284, G15-26, 2003.

[4] Nakazono, K., Watanabe, N., Matsuno, K., Sasaki, J., Sato, T., and Inoue, M.: Does superoxide underlie the pathogenesis of hypertension? Proc. Natl. Acad. Sci. U.S.A., 88, 1004510048, 1991. 
[5] Zafarullah, M., Li, W.Q., Sylvester, J., and Ahmad, M.: Molecular mechanisms of N-acetylcysteine actions. Cell Mol. Life Sci., 60, 6-20, 2003.

[6] Yan, M., Qiang, W., Liu, N., Shen, J., Lynn, W.S., and Wong, P.K.: The ataxia-telangiectasia gene product may modulate DNA turnover and control cell fate by regulating cellular redox in lymphocytes. Faseb J., 15, 1132-1138, 2001.

[7] Soga, T., Baran, R., Suematsu, M., Ueno, Y., Ikeda, S., Sakurakawa, T., Kakazu, Y., Ishikawa, T., Robert, M., Nishioka, T., and Tomita, M.: Differential metabolomics reveals ophthalmic acid as an oxidative stress biomarker indicating hepatic glutathione consumption. J. Biol. Chem., 281, 16768-16776, 2006.

[8] Kinoshita, A., Tsukada, K., Soga, T., Hishiki, T., Ueno, Y., Nakayama, Y., Tomita, M., and Suematsu, M.: Roles of hemoglobin allostery in hypoxia-induced metabolic alterations in erythrocytes: simulation and its verification by metabolome analysis. J. Biol. Chem., 282, 10731-10741, 2007.

[9] Shintani, T., Iwabuchi, T., Soga, T., Kato, Y., Yamamoto, T., Takano, N., Hishiki, T., Ueno, Y., Ikeda, S., Sakuragawa, T., Ishikawa, K., Goda, N., Kitagawa, Y., Kajimura, M., Matsumoto, K., and Suematsu, M.: Cystathionine $\beta$-synthase as a carbon monoxide-sensitive regulator of bile excretion. Hepatology, 49, 141-150, 2009.

[10] Schauer, R.J., Gerbes, A.L., Vonier, D., op den Winkel, M., Fraunberger, P., and Bilzer, M.: Induction of cellular resistance against Kupffer cell-derived oxidant stress: a novel concept of hepatoprotection by ischemic preconditioning. Hepatology, 37, 286-295, 2003.

[11] Tejima, K., Arai, M., Ikeda, H., Tomiya, T., Yanase, M., Inoue, Y., Nagashima, K., Nishikawa, T., Watanabe, N., Omata, M., and Fujiwara, K.: Ischemic preconditioning protects hepatocytes via reactive oxygen species derived from Kupffer cells in rats. Gastroenterology, 127, 14881496, 2004.

[12] Koeppel, T.A., Lehmann, T.G., Thies, J.C., Gehrcke, R., Gebhard, M.M., Herfarth, C., Otto, G., and Post, S.: Impact of $\mathrm{N}$-acetylcysteine on the hepatic microcirculation after orthotopic liver transplantation. Transplantation, 61, 13971402, 1996.

[13] Dunne, J.B., Piratvisuth, T., Williams, R., and Tredger, J.M.: Treatment of experimental ischemia/reperfusion injury with $S$-adenyosylmethionine: evidence that donor pretreatment complements other regimens. Transplantation, 63, 500-506, 1997.

[14] Nakano, H., Nagasaki, H., Barama, A., Boudjema, K., Jaeck, D., Kumada, K., Tatsuno, M., Baek, Y., Kitamura, N., Suzuki, T., and Yamaguchi, M.: The effects of Nacetylcysteine and anti-intercellular adhesion molecule-1 monoclonal antibody against ischemia-reperfusion injury of the rat steatotic liver produced by a choline-methioninedeficient diet. Hepatology, 26, 670-678, 1997.

[15] Jeon, B.R. and Lee, S.M.: $S$-adenosylmethionine protects post-ischemic mitochondrial injury in rat liver. J. Hepatol., 34, 395-401, 2001.

[16] Aruoma, O.I., Halliwell, B., Hoey, B.M., and Butler, J.: The antioxidant action of taurine, hypotaurine and their metabolic precursors. Biochem. J., 256, 251-255, 1988.

[17] Pecci, L., Montefoschi, G., Fontana, M., Dupre, S., Costa, M., and Cavallini, D.: Hypotaurine and superoxide dismutase: protection of the enzyme against inactivation by hydrogen peroxide and peroxidation to taurine. Adv. Exp. Med. Biol., 483, 163-168, 2000.

[18] Fontana, M., Giovannitti, F., and Pecci, L.: The protective effect of hypotaurine and cysteine sulphinic acid on peroxynitrite-mediated oxidative reactions. Free Radic. Res., 42, 320-330, 2008. 